\title{
A Comparative Study of Transvaginal Sonography and Pelvic MRI in Patients with Endometrial Cancer
}

\author{
Marjaneh Farazestanian1, Anahita Hamidi², Zohreh Yousefi ${ }^{*}$, Parvaneh Layegh4, Amir Hosein Jafarian5, \\ Helena Azimi ${ }^{1}$, Elham Abdollahi ${ }^{1}$ \\ ${ }^{1}$ Department of Obstetrics and Gynecology, Faculty of Medicine, Mashhad University of Medical Sciences, Mashhad, Iran \\ ${ }^{2}$ Faculty of Medicine, Mashhad University of Medical Sciences, Mashhad, Iran \\ ${ }^{3}$ Department of Obstetrics and Gynecology, Faculty of Medicine, Mashhad University of Medical Sciences, Mashhad, Iran \\ ${ }^{4}$ Department of Radiology, Faculty of Medicine, Mashhad University of Medical Sciences, Mashhad, Iran \\ ${ }^{5}$ Department of Pathology, Faculty of Medicine, Mashhad University of Medical Sciences, Mashhad, Iran
}

*Corresponding author: Zohreh Yousefi, Yousefiz@mums.ac.ir

Copyright: (C) 2022 Author(s). This is an open-access article distributed under the terms of the Creative Commons Attribution License (CC BY 4.0), permitting distribution and reproduction in any medium, provided the original work is cited.

\begin{abstract}
Background: The most prevalent type of gynecological cancer is endometrial cancer. Accurate surgical staging is the most important aspect in the management of endometrial cancer. Preoperative evaluation of myometrial invasion and appropriate management could be achieved by transvaginal sonography and pelvic MRI. Methods: A total of 53 patients with endometrial cancer, who were referred to a gynecology oncology department of an academic hospital in Mashhad University of Medical Sciences from 2018 to 2020, were evaluated in this study. Data were collected using a questionnaire on endometrial cancer. All the patients underwent preoperative transvaginal sonography and pelvic MRI to evaluate myometrial invasion. The involvement of myometrium thickness and histological findings were compared between two imaging modalities, and SPSS 23.0 was used to analyze the data. Results: Junctional irregularity was the most prevalent finding on transvaginal sonography. Based on transvaginal sonography, myometrial invasion of less than $50 \%$ was found in $73 \%$ of patients, while invasion of more than $50 \%$ was observed in $26.31 \%$. In $57.44 \%$ of MRIs, there was less than 50\% myometrial invasion, while in $42.55 \%$ of MRIs, there was more than $50 \%$ myometrial invasion. In both modalities, the most common finding was myometrial invasion of less than $50 \%$. The accuracy, sensitivity, and specificity of transvaginal sonography were $0.47,0.27$, and 0.75 , respectively, whereas the accuracy, sensitivity, and specificity of MRI were $0.54,0.45$, and 0.61 , respectively. Conclusion: MRI can be the modality of choice for evaluating myometrial invasion and optimizing endometrial cancer treatment planning, as well as reducing the complications of non-indicated lymphadenectomy.
\end{abstract}

Keywords: Endometrial cancer; Myometrial invasion; Pelvic MRI; Transvaginal sonography

Online publication: February 17, 2022

\section{Introduction}

In the United States, endometrial carcinoma (EC) is the most prevalent cancer of the female genital tract ${ }^{[1]}$. In patients with a suspicion of endometrial cancer, ultrasonography, particularly with transvaginal approach, is still the best and first imaging technique ${ }^{[2]}$. The thickness of the endometrium on transvaginal sonography is a sensitive indicator for detecting endometrial cancer in postmenopausal women. However, its sensitivity 
is estimated to be $20 \%$ lower in asymptomatic patients compared to symptomatic patients; in addition, its specificity is low (high false-positive rate). As a result, many patients still require an endometrial biopsy ${ }^{[3]}$. Transvaginal ultrasonography (TVU) is widely available in many regions around the world. It is relatively inexpensive, noninvasive, and does not use ionizing radiation. It has a high diagnostic value for detecting myometrial infiltration and cervical invasion ${ }^{[4]}$. Preoperative magnetic resonance imaging (MRI) may be useful in determining the severity of endometrial carcinoma and identifying patients with suspected nodal metastasis, which could replace surgical staging. It has been proposed that investigation via single-site laparoscopic or robotic hysterectomy with lymph node assessment, which has been attracting attention to minimize the rate of morbidity associated with EC surgery, will be of considerable importance for future directions ${ }^{[5]}$. Accurate staging of the disease at the time of diagnosis and appropriate treatment planning to reduce morbidity are two important aspects for therapeutic success ${ }^{[6]}$. The standard treatment for endometrial cancer is surgery ${ }^{[7]}$. The severity of the disease, the depth of myometrial invasion, and cervical stromal involvement are important factors in choosing the type of surgery and lymphadenectomy ${ }^{[8]}$.

In recent studies, MRI has shown to be of use in preoperative staging ${ }^{[9,10]}$. Shirvastava suggested that MRI is the best modality for preoperative staging ${ }^{[1]}$. Another research found that there is no correlation between preoperative TVS and intraoperative macroscopic examination for detecting deep myometrial invasion. Both methods showed similar accuracy when compared with frozen section histology ${ }^{[12]}$. In a study, Alcázar revealed that the diagnostic performance of TVS for detecting deep myometrial invasion in women with endometrial cancer is moderate ${ }^{[13]}$. Preoperative TVS may be considered as a method for assessing myometrial invasion as an alternative to intraoperative macroscopic examination, when performed by an experienced examiner and with appropriate imaging quality ${ }^{[12]}$.

The aim of this study was to compare transvaginal sonography and pelvic MRI to the histopathological diagnosis in patients with endometrial cancer.

\section{Methods}

A total of 53 patients with endometrial cancer, who were referred to a gynecology oncology department of an academic hospital in Mashhad University of Medical Sciences from 2018 to 2020, were evaluated in this study. Endometrial cancer was confirmed by endometrial sampling. The study was approved by the Ethics Committee of Mashhad University with the approval code of IR.MUMS.REC.328.1395. After obtaining the patients' written informed consent, they were enrolled in the study, and demographic data were collected.

A gynecologic radiologist performed transvaginal ultrasonography on all the patients. A radiological analysis was carried out using the Madison V20 machine and the $9 \mathrm{MHZ}$ vaginal probe. Preoperative pelvic contrast-enhanced MRI was also performed on all the patients using Siemens Avanto 1.5T. The MRI images were reported by an expert gynecologic radiologist. Subsequently, all the patients underwent total abdominal hysterectomy and bilateral salpingo-oophorectomy with surgical staging. A gynecologic pathologist evaluated the histopathologic outcomes of all samples. Finally, both radiologic and the histopathologic findings were compared to evaluate myometrial invasion. Statistical Package for the Social Sciences (SPSS) 23.0 was used to analyze the data. The specificity, sensitivity, and accuracy of both modalities were assessed. $P<0.05$ was considered statistically significant in all tests.

\section{Results}

The mean age of the patients was 53 years old. Patients with stage IA or IB endometrioid carcinoma in endometrial biopsy were enrolled in the study.

Cellular grading analyses in endometrial samples included grade I as the most frequent with $51.02 \%$. The least frequent was allocated to grade III with $14.28 \%$. Myometrial invasion was assessed as less or 
more than $50 \%$ of myometrial thickness in both TVS and MRI. In TVS, the endometrial line, junctional irregularity, and heterogeneity of the uterine cavity were evaluated. In non-menopausal women, the average endometrial line was noted to be $17.17 \mathrm{~mm}$, while in menopausal women, it measured $16.30 \mathrm{~mm}$. Nonparametric Mann-Whitney $U$ test showed no significant differences between menopaused and nonmenopaused cases $(P=0.134)$.

Masses in the uterine cavity were found in 24 patients $(45.3 \%)$. Analysis showed that myometrial invasion was less than $50 \%$ in $73 \%$ of cases but more than $50 \%$ in $26.31 \%$ of cases (Figure 1). The accuracy, sensitivity, and specificity of TVS were estimated to be $0.47,0.27$, and 0.75 , respectively. Data confirmed that $57.44 \%$ of cases had myometrial invasion of less than $50 \%$, but $42.55 \%$ of the cases had invasion of more than 50\%. The accuracy, sensitivity, and specificity of MRI were estimated to be $0.54,0.45$ and 0.61 , respectively (Figure 2).

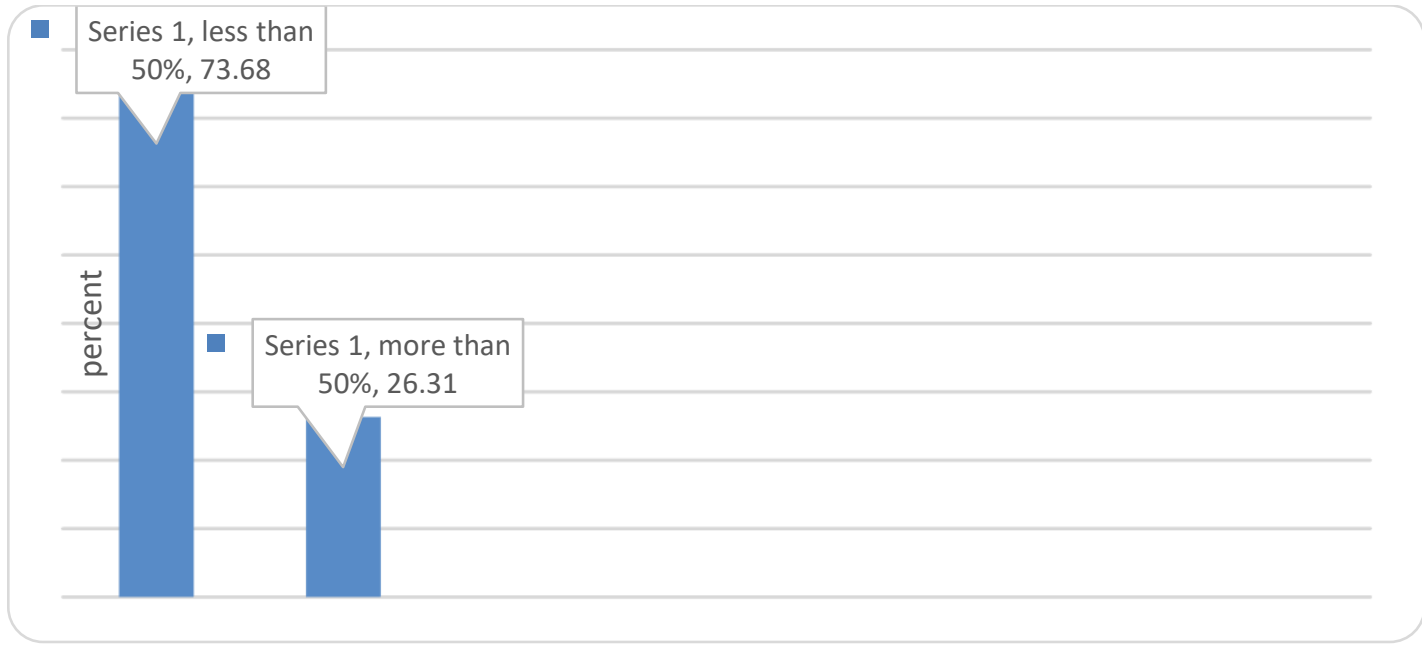

Figure 1. Frequency and distribution of myometrial invasion in TVS

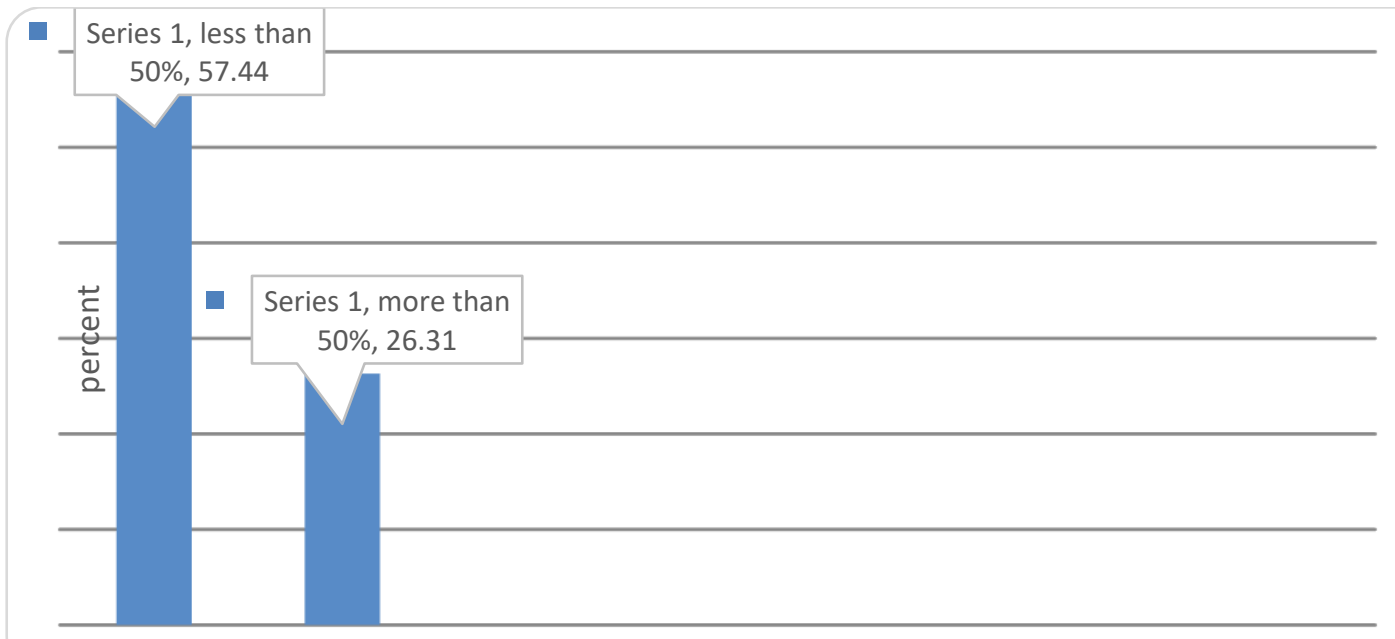

Figure 2. Frequency and distribution of myometrial invasion in MRI

According to histopathologic findings, all the patients were stage IA or IB. Grade I was the most common grade, with $67.92 \%$ of patients. In $58.49 \%$ of the cases, the myometrial invasion was less than $50 \%$, while in $41.5 \%$ of the cases, the invasion was more than $50 \%$ (Figure 3). Data analyses showed the low efficiency of curettage and final pathologic reports in cellular grading with significant Kappa value $=$ 0.018 . 


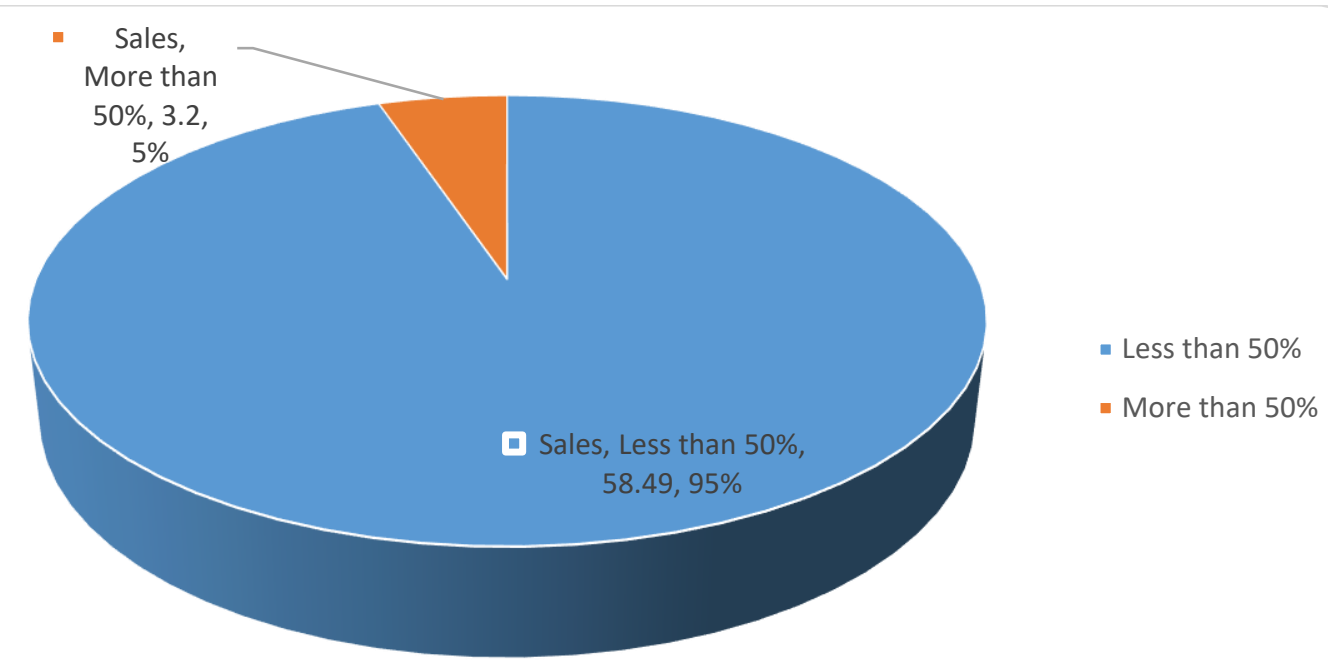

Figure 3. Frequency and distribution of myometrial invasion in pathological results

This study found that MRI is a better technique for determining myometrial invasion compared to TVS.

\section{Discussion}

The most prevalent cancer of the female reproductive system is endometrial cancer (uterine carcinoma)

${ }^{[14]}$. Endometrial cancer mostly occurs in postmenopausal women and is associated with obesity ${ }^{[15]}$.

Many factors affect the prognosis and management of uterine carcinoma, including the behaviour of the tumor, the degree myometrial infiltration, and presence of lymphatic or hematogenous dissemination ${ }^{[14]}$. The majority of endometrioid carcinomas are of low grade (grade 1 or 2) and are diagnosed early, with good prognosis ${ }^{[16]}$. Fortunately, the majority of women with endometrial cancer survive their first diagnosis, making them the most common kind of gynecologic cancer survivors ${ }^{[3]}$. For example, in the United States, there was an estimate of 807,860 survivors with a history of uterine cancer in $2019^{\text {[17] }}$. It has been shown that the depth of myometrial invasion is directly associated with lymph node involvement and the prognosis of the patients with endometrial cancer ${ }^{[7]}$. Avoiding unnecessary lymphadenectomy is important, especially for low-risk patients, based on the depth of myometrial invasion, grading, and histological subtype prior to surgery ${ }^{[18]}$. Currently, TVS and MRI are the commonest techniques used for assessing the depth of myometrial invasion preoperatively . ${ }^{[19]}$ At present, MRI is recommended for preoperative imaging in several guidelines ${ }^{[20]}$. The accuracy, sensitivity, and specificity of endometrial MRI were estimated to be $0.54,0.45$, and 0.61 , respectively ${ }^{[11]}$. In Shrivastava's study, myometrial invasion of less than $50 \%$ was found in $16 \%$ of patients, MI of more than $50 \%$ was found in $55 \%$ of patients, and total myometrial invasion was found in $5 \%$ of the cases ${ }^{[11]}$. In a study conducted by Gallego and other researchers, among 51 women diagnosed with endometrial cancer, the sensitivity and specificity of MRI were estimated to be $90 \%$ and $77.8 \%$, respectively ${ }^{[18]}$. In Savellie's study, the sensitivity and specificity were estimated to be $75 \%$ and $89 \%$, respectively ${ }^{[21]}$. In another study, MRI showed better sensitivity than TVS for detecting deep myometrial invasion in women with endometrial cancer; however, the difference observed was not statistically significant ${ }^{[13]}$. In the aforementioned study, myometrial invasion of less than $50 \%$ was detected in $58.49 \%$ of the cases, while invasion of more than $50 \%$ was noted in $41.5 \%$ of the patients ${ }^{[22]}$. Shrivastava found that grade I endometrioid adenocarcinoma was the most prevalent histopathology and proposed that contrast-enhanced MRI is the modality of choice in the preoperative staging of endometrial cancer ${ }^{[11]}$. Dul and other researchers suggested that MRI provides a high degree of accuracy in the diagnosis and evaluation of myometrial and deep myometrial invasion ${ }^{[23]}$. This method is also suitable for predicting preoperative 
staging of endometrial carcinoma. MRI is a useful method for assisting clinicians in making decisions about the best treatment options ${ }^{[23]}$. In a study, it was indicated that the sensitivity, specificity, positive predictive value, negative predictive value, and accuracy of transvaginal ultrasound in detecting the invasion of myometrial cavity were $66.25 \%, 75.0 \%, 90.00 \%, 30.00 \%, 68.00 \%$, respectively, whereas in distant metastasis, the variables were $77.78 \%, 80.95 \%, 63.64 \%, 89.47 \%$, and $80 \%$, respectively [24]; the aforementioned variables for MRI were $88.89 \%, 100.00 \%, 100.00 \%, 66.67 \%$, and $90.91 \%$, respectively, whereas in distant metastasis, the respective variables were all $100.00 \%{ }^{[24]}$.

In regard to intraoperative frozen section, the sensitivity and specificity were $90 \%$ and $73 \%$, respectively, in a study. In comparison with MRI findings, the study showed that MRI with appropriate tumor mapping has the same accuracy as intraoperative histopathological studies by frozen section ${ }^{\text {[23] }}$.

The notion that an experienced radiologist evaluated and reported the MRI images is a positive feature of this study. However, the small sample size is a limitation of this study. As MRI is expensive and has limited availability, many studies have chosen to use other modalities, such as color Doppler sonography or dynamic contrast-enhanced MRI. In the treatment of endometrial cancer, these modalities help to minimize problems caused by non-indicated lymphadenectomy.

In conclusion, TVUS has shown to be a useful modality for the measurement of endometrial thickness. It is practical, inexpensive, and an easy imaging procedure. It was the preferred choice for screening of patients with suspicion of EC, especially when conducted by a skilled radiologist. MRI is an effective and useful method for assessing myometrial invasion and optimal treatment planning of endometrial cancer, with possibility of reducing the complications of non-indicated lymphadenectomy.

\section{Acknowledgment}

The authors would like to extend their gratitude to the staffs in the Department of Gynecology Oncology at Mashhad University of Medical Sciences for supporting and funding the study. They are also grateful to the patients for collaborating and participating in this study.

\section{Disclosure statement}

The authors declare that this study is a terminated student research project (code: 931 762) with conflicts of interest with the University Research Center of Mashhad University of Medical Sciences.

\section{References}

[1] Guerriero S, Saba L, Pascual MA, et al., 2018, Transvaginal Ultrasound Vs Magnetic Resonance Imaging for Diagnosing Deep Infiltrating Endometriosis: Systematic Review and MetaAnalysis. Ultrasound Obstet Gynecol, 51(5): 586-595.

[2] O’Malley R, Dighe MK, 2014, Invited Commentary on American College of Radiology Appropriateness Criteria Endometrial Carcinoma. Ultrasound Quarterly, 30(1): 29-31.

[3] Tarney CM, Wang G, Bateman NW, et al., 2019, Biomarker Panel for Early Detection of Endometrial Cancer in the Prostate, Lung, Colorectal, and Ovarian Cancer Screening Trial. Am J Obstet Gynecol, 221(5): 472.

[4] Lalwani N, Dubinsky T, Javitt MC, et al., 2014, ACR Appropriateness Criteria Pretreatment Evaluation and Follow-Up of Endometrial Cancer. Ultrasound Quarterly, 30(1): 21-28.

[5] Corrado G, Calagna G, Cutillo G, et al., 2018, The Patient and Observer Scar Assessment Scale to Evaluate the Cosmetic Outcomes of the Robotic Single-Site Hysterectomy in Endometrial Cancer. Int 
J Gynecol Cancer, 28(1): 194-199.

[6] Miklos P, Klacko M, Babala P, et al., 2014, Transvaginal Ultrasound Examination of Myometrial Infiltration by Endometrial Cancer. Bratisl Lek Listy, 115(1): 14-18.

[7] Zamani F, Goodarzi S, Hallaji F, et al., 2012, Diagnostic Value of Pelvic MRI for Assessment of the Depth of Myometrial Invasion and Cervical Involvement in Endometrial Cancer: Comparison of New Versus Old FIGO Staging. Iran J Radiol, 9(4): 202-208.

[8] Aryan A, Askari F, Mohammadifar M, et al., 2016, Role of MRI (Magnetic Resonance Imaging) in Preoperative Staging of Endometrial Cancer: A Review. Austin J Radiol, 3(1): 1045.

[9] Crispens M, 2016, Endometrial Cancer, in Rock J, Jones H, Telinde's Operative Gynecology, 10th Edition, Wolters \& Kluwer, Philadelphia, 1290-1295.

[10] Anciaux D, Lawrence W, 1997, Malignant Uterine Tumors: An overview, in Shingleton H, Fowler W, Jordan J et al., Gynecology Oncology: Current Diagnosis and Treatment, 1st Edition, Saunders, Philadelphia, 130-143.

[11] Shrivastava S, Barmon D, Kataki AC, et al., 2016, Magnetic Resonance Imaging in Pre-Operative Staging of Endometrial Cancer. Indian J Cancer, 53(1): 181-185.

[12] Pineda L, Alcázar JL, Caparros M, et al., 2016, Agreement between Preoperative Transvaginal Ultrasound and Intraoperative Macroscopic Examination for Assessing Myometrial Infiltration in Low-Risk Endometrioid Carcinoma. Ultrasound Obstet Gynecol, 47(3): 369-373.

[13] Alcázar JL, Orozco R, Martinez-Astorquiza CT, et al., 2015, Transvaginal Ultrasound for Preoperative Assessment of Myometrial Invasion in Patients with Endometrial Cancer: A Systematic Review and Meta-Analysis. Ultrasound Obstet Gynecol, 46(4): 405-413.

[14] Jiang J, Zhao JL, Qing JF, et al., 2018, Endometrial Carcinoma: Diffusion-Weighted Imaging Diagnostic Accuracy and Correlation with Ki-67 Expression. Clinical Radiology, 73(4): 413.

[15] Arthur RS, Kabat GC, Kim MY, et al., 2019, Metabolic Syndrome and Risk of Endometrial Cancer in Postmenopausal Women: a Prospective Study. Cancer Causes \& Control, 30(4): 355-363. DOIL 10.1007/s10552-019-01139-5

[16] Singh N, Hirschowitz L, Zaino R, et al., 2019, Pathologic Prognostic Factors in Endometrial Carcinoma (Other Than Tumor Type and Grade). Int J Gynecol Pathol, 38 Suppl 1(1 Suppl 1): S93S113.

[17] Miller KD, Nogueira L, Mariotto AB, et al., 2019, Cancer Treatment and Survivorship Statistics. CA Cancer J Clin, 69(5): 363-385.

[18] Gallego JC, Porta A, Pardo MC, et al., 2014, Evaluation of Myometrial Invasion in Endometrial Cancer: Comparison of Diffusion-Weighted Magnetic Resonance and Intraoperative Frozen Sections. Abdom Imaging, 39(5): 1021-1026.

[19] Lee YJ, Moon MH, Sung CK, et al., 2016, MR Assessment of Myometrial Invasion in Women with Endometrial Cancer: Discrepancy between T2-Weighted Imaging and Contrast-Enhanced T1Weighted Imaging. Abdom Radiol (NY), 41(1): 127-135.

[20] Sundar S, Balega J, Crosbie E, et al., 2017, BGCS Uterine Cancer Guidelines: Recommendations for Practice. Eur J Obstet Gynecol Reprod Biol, 213: 71-97.

[21] Savelli L, Testa AC, Mabrouk M, et al., 2012, A Prospective Blinded Comparison of the Accuracy of Transvaginal Sonography and Frozen Section in the Assessment of Myometrial Invasion in Endometrial Cancer. Gynecol Oncol, 124(3): 549-552. 
[22] Alcazar JL, 2017, Transvaginal Ultrasound Versus Magnetic Resonance Imaging for Preoperative Assessment of Myometrial Infiltration in Patients with Endometrial Cancer: A Systematic Review and Meta-Analysis. J Gynecol Oncol, 28(6): e86.

[23] Du L, Lei Y, Li D, et al., 2012, Value of Magnetic Resonance Imaging in Preoperative Staging of Endometrial Carcinoma According to International Federation of Gynecology and Obstetrics (2009) Staging Criteria. Nan Fang Yi Ke Da Xue Xue Bao, 32(7): 1048-1051.

[24] Mohamed DM, El-Wahab abo Dewan KA, Mera SM, 2018, The Diagnostic Accuracy of Transvaginal Ultrasonography, Magnetic Resonance Imaging and Diffusion Weighted Image in Female Patients with Endometrial Carcinoma. Alexandria Journal of Medicine, 2018: 685-691. DOI: 10.1016/j.ajme.2018.10.002 